\title{
PROBLEMAS CON EL ASPECTO VERBAL EN E/LE
}

\author{
Santiago Roca Marín \\ Universidad de Alicante \\ Santiago.Roca@ua.es
}

A los alumnos europeos que llegan a nuestras universidades no les resulta fácil deslindar entre tiempo y aspecto verbal. Éstos alumnos vienen para aprender español o perfeccionarlo a la vez que realizan un curso de la carrera en que están matriculados en su país de origen. Para muchos de ellos, el aspecto es una unidad o un matiz que no consiguen ver claro. Suelen tener menos problemas como receptores que como hablantes en español. En el segundo caso, como hablantes, unen el uso del aspecto a un «marcador textual»; en ausencia de este, la duda aparece y con ella la vacilación en la forma verbal.

Con este trabajo se ha querido corroborar de una forma estadistica esta vacilación y confusión en la utilización del aspecto flexivo, la «oposición de formas de un mismo verbo» (Miguel, E.:1999, 2992), que se percibe en las aulas ordinarias donde hay alumnos del Programa Sócrates en la Universidad de Alicante.

En colaboración con el Servicio de Relaciones Internacionales de la Universidad de Alicante donde reciben clase de español para extranjeros estos alumnos, se ha procedido a realizar una prueba al nivel intermedio y avanzado de E/LE durante noviembre de 2003 . El númcro de alumnos encuestados suma un total de 110. Estos alumnos están divididos en dos grupos: a) nivel medio, 90 alumnos; b) nivel avanzado, 20 alumnos. Previamente, la prueba fue realizada a 20 encuestados cuya lengua materna era el español para detectar las posibles variaciones sobre algún ítem.

Se divide, pues, este trabajo en dos partes; una primera sería el marco teórico y el tratamiento que del mismo hacen los libros de tcxto, en concreto una selección de los mismos. Una segunda parte, sería el análisis de la prueba realizada a los alumnos sócrates de la Universidad de Alicante en el primer cuatrimestre del curso académico 2003/2004.

\section{REVISIÓN TEÓRICA EN LOS MANUALES DE E/LE}

\subsection{Marco teórico}

Antes de entrar en el análisis teórico y práctico que realizan los libros de texto que hemos analizado sobre cl aspecto, dcbemos definir qué entendemos por tal.

En este trabajo analizamos el aspecto flexivo (Elena de Miguel: 1999) en un sentido prototípico (Graciela Reyes: 1995) y semántico, aunque no podemos obviar ni olvidar el 
valor pragmático y la modalización que se produce en el aspecto como señala Graciela Reyes (1995).

Entendemos, como Elena de Miguel (1999: 2998), que «el 'aspecto flexivo' es la información relativa al modo en que tiene lugar un evento que viene proporcionada por los morfemas flexivos del verbor. De esta manera se va a estudiar en los manuales de E/LE que hemos cotejado y posteriormente en la correcta utilización que hacen los alumnos sócrates del aspecto ficxivo scgún al evento al que se relaciona.

EI otro accidente con el que confunden los alumnos de español para extranjeros el aspecto es el tiempo, siguiendo la definición de Elcna de Miguel (1999:2989)

el 'tiempo' es una categoria deíctica: localiza el evento verbal en un ticmpo externo, oricntándolo bien en relación con $\mathrm{cl}$ momento de habla, bien en relación con el tiempo en que ticne lugar otro evento. El aspecto, en cambio, se ocupa del tiempo como una propiedad inherente $o$ interna del propio evento: muestra el evento tal y como este se desarrolla 0 distribuye en el tiempo, sin hacer referencia al momento del habla.

Es en esta dicotomía que plantea ambas definiciones, lo externo y lo interno al evento, donde radica gran parte de la dificultad para la utilización de los valores aspectuales en español por parte de los extranjeros. Esto se acentúa sobre todo cuando no hay un rasgo morfológico que permita reconocer el uso o utilización del aspecto, sólo en los enunciados donde aparecen marcadores temporales lo reconocen y no siempre de una forma clara.

En este trabajo no se analiza el uso de la aspectualidad en un sentido amplio, es decir, el aspecto flexivo y el léxico. Sólo analizaremos el uso del aspecto flexivo. Existen otros trabajos donde se ha intentado ver el uso del aspecto léxico on alumnos cxtranjeros (Matínez Bazán, A.: 1994).

Por último, nos quedaría hablar de los marcadores, anclajes a los que nos referiamos y que la mayoría de los libros de E/LE utilizan como elementos cuyo conocimiento, en muchos casos, permite un uso inequívoco del aspecto. Graciela Reyes dice de ellos que

«hay diferentes tipos de marcadores pragmáticos: los shifters como aquí, ayer, etc., que relacionan un enunciado con su contexto extralingüistico» (Reyes, G.:1990; 23)

Matte Bon, F. (1995) en su gramática de español para extranjeros establece la oposición del aspecto flexivo entre indefinido/ perfecto/ imperfecto de la siguiente forma:

la oposición pretérito indefinido / pretérito perfecto (pasado en el presentc) es algo exquisitamente lingüístico, que se da $\mathrm{cn} \mathrm{cl} \mathrm{nivel} \mathrm{en}$ cl que la lengua remite a sí misma y no en su dimensión referencial. Si se intenta a toda costa encontrar en el mundo real del que hablamos con la lengua una oposición que en él no existe, se caerá inevitablemente, en aproximaciones y errores como creer que el pretérito imperfecto (pasado en el presente) se refiere a acciones más recientes. No hay que buscar esta oposición en las acciones y los acontecimientos extralinguísticos en sí, sino en lo que hace el hablante con ellos al evocarlos lingüísticamente:

- Con el pretérito indefinido, los [los acontecimientos] presenta como centro de su interés.

- Con el imperfecto, los utiliza para crear un marco contextual, evocar una situación (los hechos ya no interesan en si, sino tan sólo en la medida en que crean una situación) 
- Con el pretérito perfecto (pasado en el presente), el enunciador habla de cosas pasadas que le interesan por su relación con el presente de la enunciación: sigucn vigentes, explican el presente, etc. (Matte Bon, 1995; 115)

Lo más significativo de los libros de texto de E/LE es que señalan la utilización del aspecto en relación a marcadores temporales en última instancia, aunque previamente describan los usos y funciones.

\subsection{Uso en los libros de texto}

Partiendo de la definición de aspecto y de los distintos modos de expresar el aspecto flexivo, vamos a analizar cómo lo reflejan algunos libros de texto de E/LE.

a) INDEFINIDO:

En Sueña $(2000 ; 138)$ se dice del indefinido que usirve para narrar hechos concretos del pasado» y añade una serie de marcadores textuales con los que se relaciona.

En Prisma A2 (2003: 38) sc dice del indefinido:

- Se utiliza para expresar acciones pasadas en un periodo de tiempo terminado

- Para expresar acciones pasadas de desarrollo prolongado, pero limitado y cerrado

- Para expresar acciones que se han repetido en el pasado

$\mathrm{Y}$ añade una serie de marcadores temporales con los que se relaciona. Estos marcadores juntos con los de Sueña aparecen en el siguiente cuadro:

\begin{tabular}{|l|l|}
\hline \multicolumn{2}{|c|}{ MARCADORES TEMPORALES CON INDEFINIDO } \\
\hline Prisma A2:2003, 38 y 39 & Sueña $1: 2000,117$ y 118 \\
\hline Anoche / Ayer / Anteanoche / El otro dia & Ayer / Anteayer/ \\
Hacc dos días/ semanas/ tres meses.... & La semana pasada \\
El mes pasado / año/ verano...pasado & Hace......... \\
En 1990/ agosto / verano & Este año \\
& Aquel día \\
& El verano pasado \\
& En 1992 \\
\hline
\end{tabular}

Sin embargo, en cuanto el alumno se enfrenta a un texto donde apenas aparecen marcadores temporales, comienza a dudar. Identifica tiempo y aspecto como una unidad. En el ejercicio que les propusimos, se centraba mayoritariamente en el aspecto y no en el tiempo, se intentaba analizar cl dominio idiomático del aspecto eliminando en la medida de lo posible los marcadores textuales. El resultado que analizaremos en la tcrcera parte de este trabajo resultó bastante significativo y confirmó lo que en un principio pensábamos sobre las dudas que les surgen a los alumnos extranjeros en el uso y frecuencia del aspecto cuando no aparece unido a marcadores temporales. 
b) PRETÉRITO PERFECTO:

Matte Bon dice del pretérito perfecto que

en realidad se trataba más de un tiempo del presente que del pasado, ya que es una manera de hablar de cosas pasadas, presentándolas como algo que, en el contexto considerado, no nos interesa en sí, sino en su relación con el presente (Matte Bon, F 1995: 114)

En el libro Sueña 1 (2000: 103) dice del pretérito perfecto:

uso: es el pasado del presente. Expresa una acción pasada que el hablante siente cercana al momento presente

Esta definición que utilizan los autores de Sueña 1 emplea a la vez el «cercana» y «presente» pero no coincide en el elemento tan significativo que señala Matte Bon «[hecho del pasado que interesa] en su relación con el presente» $(1995,114)$. El adjetivo «cercana» genera una ambigüedad para el estudiante, un uso arbitrario que puede conducir más al error al alumno que a una clarificación del hecho que se pretende aclarar.

En Prisma A2 define el pretérito perfecto como:

Este tiempo se utiliza principalmente para describir o narrar lo que ha pasado/ has hecho:

1. Describir o narrar accioncs pasadas en cl periodo de un tiempo no terminado

2. Describir o narrar acciones pasadas en un periodo de tiempo terminado que el hablante considera cercano al presente (pasado 'reciente')

3. Para expresar la realización o no de un hecho en el pasado (hasta el presente) y su frecuencia. (Prisma A2: 2003, 49)

Libros como Prisma $A 2$ o $A U L A 2$ que son de reciente publicación siguen estableciendo la explicación del pretérito perfecto en relación a marcadores temporales.

En el siguicntc cuadro podemos observar los marcadores temporales que señalan los manuales Prisma A2 y Sueña 1 para identificar el uso del pretérito perfecto.

Añade: siempre, toda mi vida, muchas veces varias veces, algunas veces, $n^{0}$ de veces, ninguna vez, nunca, en la vida jamás (Prisma A2: 2003, 49):

El manual $A U L A 2$ (2003), Edit. Difusión, en la página 84 del libro del alumno en cl ejercicio $4, A$ y $B$ establece una serie de marcadores relacionados con el pretérito perfecto y el pretérito indefinido: hoy, esta mañana, ayer, esta semana, el año pasado, nunca, el lunes, en el año 1999, iltimamente para que el alumno los clasifique en relación con el pretérito perfecto o indefinido. Este ejercicio lo repite varias veces.

El uso de un modelo comunicativo puede hacer que el autor de un libro de E/LE tenga que plantearse qué elementos externos pueden ayudar al futuro alumno en los procesos comunicativos en los que tenga que utilizar el aspecto. Pero sin una reflexión gramatical y pragmática, que obvian la mayoría de los libros cotejados, al alumno le resulta difícil esta distinción.

Un modelo comunicativo es útil pero utilizando, en algunos casos, como refuerzo un método gramatical o como señala García Santa-Cecilia (1995) un método gramatical comunicativo; si no, corremos el riesgo de que el alumno intente sistematizarlo, en la mayoría de 


\begin{tabular}{|c|c|c|c|}
\hline \multicolumn{2}{|c|}{ Prisma A2 $(2003,49)$} & & Sueña 1 \\
\hline Este & $\begin{array}{l}\text { Fin de semana } \\
\text { Mes } \\
\text { Trimestre } \\
\text { Semestre } \\
\text { Año } \\
\text { Verano } \\
\text { Otoño } \\
\text { invierno }\end{array}$ & \multirow[t]{2}{*}{$\begin{array}{l}\text { Pese a que en ambos manuales estos marcadores } \\
\text { temporales se relacionan con el pretérito perfecto } \\
\text { compuesto, también pueden se utilizados con el } \\
\text { perfecto simple, ej: } \\
\text { Hoy comí paella }\end{array}$} & \multirow[t]{2}{*}{$\begin{array}{l}\text { Iloy } \\
\text { Esta mañana } \\
\text { Últimamente } \\
\text { Estc fin de } \\
\text { scmana } \\
\text { Este año } \\
\text { Este curso }\end{array}$} \\
\hline Esta & $\begin{array}{l}\text { Tarde } \\
\text { Mañana } \\
\text { Semana } \\
\text { primavera }\end{array}$ & & \\
\hline Hace & $\begin{array}{l}\text { Menos de } 24 \text { horas } \\
\text { Un instante } \\
10 \text { minutos } \\
\text { un rato } \\
\text { un momento }\end{array}$ & $\begin{array}{l}\text { Aquí cl uso del pretérito perfecto coincide con los } \\
\text { marcadores, pero, } \\
\text { Ej. con indefinido: } \\
\text { Hace ya un rato que comí }\end{array}$ & $\begin{array}{l}\text { Hace un rato } \\
\text { Hace tres meses }\end{array}$ \\
\hline
\end{tabular}

los casos como algo simplemente temporal, comparándolo con su propia lengua, y esto se enquiste pasando a formar parte de la interlengua del alumno.

c) IMPERFECTO:

El manual Prisma A2 dice del imperfecto que

Con él nos referimos al contexto, describimos las circunstancias o las acciones habituales del pasado (Prisma A2: 2003, 105)

En Sueña $1(2000,132)$ de Anaya, se describe los usos del pretérito imperfecto:

1. Hablar de costumbres y hábitos en el pasado

2. Describir personas, lugares y cosas dentro de un contexto de pasado

3. Describir las situaciones o los contextos de las acciones

\begin{tabular}{|l|}
\hline \multicolumn{1}{|c|}{ MARCADORES TEMPORALES CON EL IMPERFECTO } \\
\hline \multicolumn{1}{|c|}{ Sueña1: 2000,128 } \\
\hline A los docc años.... \\
De pequeño..... \\
De joven..... \\
Cuando.... \\
Antes.......hora \\
Ayer...........hoy \\
\hline
\end{tabular}


Cuando puede ser interpretado como un marcador temporal de frecuencia y por tanto introduce el imperfecto o de narración y entonces introduce el indefinido, ej.:

En la página 127 y 128 de Sueñal aparecen ejercicios con «cuando era pequeño......» «Cuando tenía doce años, me enfadaba mucho con mi hermana» (Sueñal, 2000; 132)

En la página 118 del mismo libro dentro del «esquema gramatical» en «expresar el tiempo» apartado 2 «Construcciones temporales» aparece Cuando + indefinido: «cuando cumplió 22 años se trasladó a Roma».

Esto explicaría la duda que se genera sobre todo en el nivel avanzado la propuesta del texto: Cuando [12]salimos del restaurante nos [13]encontramos con Mónica:

Nivel medio

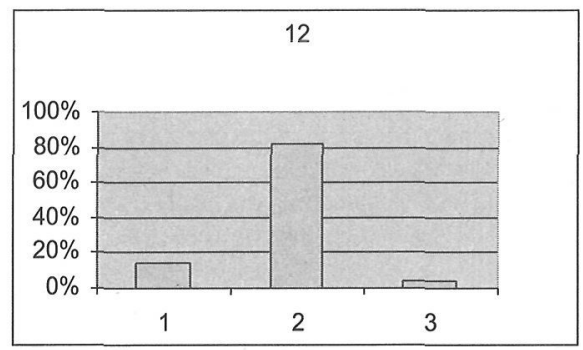

Nivel avanzado

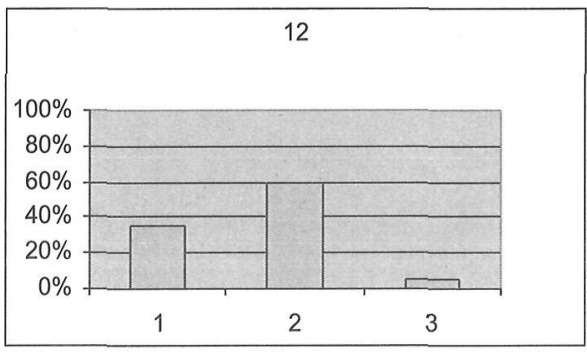

Donde la opción 1 de la tabla correspondería al pretérito imperfecto, la 2 al pretérito indefinido y la 3 al pretérito perfecto.

\section{ANÁLISIS DE RESULTADOS}

\subsection{Descripción de la prueba}

La prueba consistía, dado un texto en el que los verbos iban en infinitivo (anexo I), contestar a un cuestionario con tres opciones en cada pregunta donde aparecían la forma verbal del infinitivo de dicho texto en pretérito imperfecto de indicativo, pretérito indefinido de indicativo y pretérito perfecto de indicativo para que el alumno optara por una de las tres opciones en cada uno de los verbos que debían ser sustituidos. En la prueba que se les pasó al alumnado 
sólo aparecían tres marcadores temporales del tipo analizado en el segundo apartado de este trabajo: el fin de semana, cuando, el sábado. Estos marcadores permitian al alumno anclar el texto en unas coordenadas espacio-temporales a partir de las cuales se iría estableciendo una relación aspectual desde el presente de la enunciación. Reconocemos, pues, la dificultad del ejercicio en la que la voz del emisor podría optar por una u otra forma; en la mayoría, sólo por una, pero de lo que se trataba era de demostrar la dificultad añadida que para un hablante extranjero tiene la utilización adecuada del aspecto. Para más de un gramático es un elemento de rango secundario, pero en la articulación del discurso tiene un papel relevante.

Los matices que para un hispanohablante distinguidor de las formas imperfecto, perfecto simple y perfecto compuesto aporta cl aspecto queda reducido en estos alumnos extranjeros sócrates como hemos podido constatar en la encuesta que se les pasó.

Toda prueba, por muy objetiva que sea, plantea elementos subjetivos y en lo referente al aspecto lingüístico parece que más. Como homos dicho anteriormente, cabría interpretación por parte de los hispanohablantes en algunos de los ílems, sobre todo en las zonas dialectales donde el hablante opta por una forma u otra de perfecto, pero en la mayoría de los casos estarían de acuerdo. Para evitar esta subjetividad, previamente se pidió a veinte personas adultas de nivel educativo alto que realizaran la prueba para descartar algún itcm que planteara problemas. Los resultados de esta encuesta previa fueron satisfactorios por lo que se dio por válida para pasarla a los alumnos extranjeros.

Como todo texto está sujeto al pensamiento del emisor, autor, aquel que ha realizado el mismo, las referencias pragmáticas del discurso pertenecen al emisor. Entendemos que el alumno, que no tiene una alta competencia pragmática en español, pueda desconocer elementos pragmáticos que el autor del texto ha tenido en cuenta a la hora de realizarlo, pero también entendemos que el contexto del enunciado da las claves suficientes que subsamen esta posible ambigüedad interpretativa. Otra cosa es lo que intentamos demostrar con la prueba, la dificultad de un hablante extranjero para dominar el aspecto flexivo en español y la limitación de los manuales de lengua de no enseñarlo fuera del anclaje que supone, en la mayoría de los casos, los marcadores temporales.

\subsection{Descripción del alumnado}

La prueba fue realizada por alumnos sócrates en el primcr cuatrimestre de estancia en España. Estos alumnos estudiaban español para extranjeros en la Sociedad de relaciones internacionales de la Universidad de Alicante y estaban matriculados en un curso de nivel medio. La prueba fue realizada sin previo aviso y no estaban en ese periodo cstudiando el aspecto verbal en español. Estos alumnos llcgan a la universidad para realizar un una estancia de un cuatrimestrc o dos y cursar los estudios universitarios que debcrian hacer en su país de origen, cualquiera de los 15 países de la UE, hablamos antes del 1 de mayo de 2004. Son alumnos que se matriculan en E/LE a la misma vez que van a clase ordinaria dentro de su carrera. Fl input al que están expuestos estos alumnos es el de un nivel formal en español; apartc del que puedan establecer en sus relaciones con alumnos españoles, aunque a veces y con frecuencia se suelen relacionar entre los alumnos de un mismo país después de las clases. En algunos casos, estos alumnos sólo están en contacto con el español en las clases de E/LE o en las clases ordinarias de la universidad. En el resto del tiempo, su interacción en lengua españoła es mínima. 


\subsection{Análisis de la prueba}

Esta primera columna corresponde a los resultados porcentuales de la encuesta de los alumnos de nivel medio. Correspondiendo, como hemos dicho anteriormente, los números de la parte superior horizontal a una de las tres opciones: 1 imperfecto, 2 pretérito indefinido, 3 pretérito perfecto. La columna número 4 corresponde a la utilización del perfecto flexivo en el texto original, es decir, la respuesta que se esperaba del encuestado. La numcración de la primera columna correspondería a cada uno de los ítems.

\begin{tabular}{|c|c|c|c|c|}
\hline & 1 & 2 & 3 & 4 \\
\hline 1 & $2 \%$ & $97 \%$ & $1 \%$ & 2 \\
\hline 2 & $87 \%$ & $7 \%$ & $7 \%$ & 1 \\
\hline 3 & $6 \%$ & $84 \%$ & $10 \%$ & 2 \\
\hline 4 & $3 \%$ & $90 \%$ & $7 \%$ & 2 \\
\hline 5 & $22 \%$ & $45 \%$ & $31 \%$ & 3 \\
\hline 6 & $9 \%$ & $56 \%$ & $35 \%$ & 2 \\
\hline 7 & $14 \%$ & $65 \%$ & $21 \%$ & 3 \\
\hline 8 & $7 \%$ & $52 \%$ & $41 \%$ & 3 \\
\hline 9 & $17 \%$ & $43 \%$ & $40 \%$ & 3 \\
\hline 10 & $83 \%$ & $13 \%$ & $5 \%$ & 1 \\
\hline 11 & $75 \%$ & $18 \%$ & $7 \%$ & 1 \\
\hline 12 & $15 \%$ & $82 \%$ & $3 \%$ & 2 \\
\hline 13 & $11 \%$ & $77 \%$ & $11 \%$ & 2 \\
\hline 14 & $40 \%$ & $19 \%$ & $41 \%$ & 1 \\
\hline 15 & $8 \%$ & $88 \%$ & $5 \%$ & 2 \\
\hline 16 & $14 \%$ & $78 \%$ & $8 \%$ & 2 \\
\hline 17 & $79 \%$ & $17 \%$ & $4 \%$ & 1 \\
\hline 18 & $79 \%$ & $15 \%$ & $7 \%$ & 1 \\
\hline 19 & $15 \%$ & $74 \%$ & $11 \%$ & 3 \\
\hline 20 & $46 \%$ & $48 \%$ & $6 \%$ & 1 \\
\hline 21 & $44 \%$ & $48 \%$ & $8 \%$ & 3 \\
\hline 22 & $60 \%$ & $15 \%$ & $26 \%$ & 3 \\
\hline 23 & $5 \%$ & $75 \%$ & $20 \%$ & 3 \\
\hline 24 & $82 \%$ & $11 \%$ & $7 \%$ & 1 \\
\hline 25 & $55 \%$ & $35 \%$ & $10 \%$ & 3 \\
\hline 26 & $80 \%$ & $11 \%$ & $8 \%$ & 1 \\
\hline 27 & $48 \%$ & $27 \%$ & $25 \%$ & 3 \\
\hline 28 & $7 \%$ & $57 \%$ & $36 \%$ & 3 \\
\hline 29 & $13 \%$ & $47 \%$ & $40 \%$ & 2 \\
\hline 30 & $33 \%$ & $31 \%$ & $36 \%$ & 3 \\
\hline
\end{tabular}


Esta segunda columna corresponde a los resultados porcentuales de la encuesta de los alumnos de nivel avanzado.

\begin{tabular}{|c|c|c|c|c|}
\hline & 1 & 2 & 3 & 4 \\
\hline 1 & $5 \%$ & $85 \%$ & $10 \%$ & 2 \\
\hline 2 & $80 \%$ & $15 \%$ & $5 \%$ & 1 \\
\hline 3 & $5 \%$ & $90 \%$ & $5 \%$ & 2 \\
\hline 4 & $10 \%$ & $65 \%$ & $25 \%$ & 2 \\
\hline 5 & $30 \%$ & $35 \%$ & $35 \%$ & 3 \\
\hline 6 & $5 \%$ & $65 \%$ & $30 \%$ & 2 \\
\hline 7 & $15 \%$ & $55 \%$ & $30 \%$ & 3 \\
\hline 8 & $15 \%$ & $25 \%$ & $60 \%$ & 3 \\
\hline 9 & $10 \%$ & $50 \%$ & $40 \%$ & 3 \\
\hline 10 & $70 \%$ & $20 \%$ & $10 \%$ & 1 \\
\hline 11 & $60 \%$ & $25 \%$ & $15 \%$ & 1 \\
\hline 12 & $35 \%$ & $60 \%$ & $5 \%$ & 2 \\
\hline 13 & $5 \%$ & $70 \%$ & $25 \%$ & 2 \\
\hline 14 & $30 \%$ & $20 \%$ & $50 \%$ & 1 \\
\hline 15 & $10 \%$ & $75 \%$ & $15 \%$ & 2 \\
\hline 16 & $20 \%$ & $60 \%$ & $20 \%$ & 2 \\
\hline 17 & $95 \%$ & $5 \%$ & $0 \%$ & 1 \\
\hline 18 & $80 \%$ & $20 \%$ & $0 \%$ & 1 \\
\hline 19 & $10 \%$ & $90 \%$ & $0 \%$ & 3 \\
\hline 20 & $45 \%$ & $40 \%$ & $15 \%$ & 1 \\
\hline 21 & $70 \%$ & $25 \%$ & $5 \%$ & 3 \\
\hline 22 & $70 \%$ & $15 \%$ & $15 \%$ & 3 \\
\hline 23 & $0 \%$ & $90 \%$ & $10 \%$ & 3 \\
\hline 24 & $60 \%$ & $40 \%$ & $0 \%$ & I \\
\hline 25 & $50 \%$ & $30 \%$ & $20 \%$ & 3 \\
\hline 26 & $85 \%$ & $15 \%$ & $0 \%$ & 1 \\
\hline 27 & $60 \%$ & $15 \%$ & $25 \%$ & 3 \\
\hline 28 & $0 \%$ & $65 \%$ & $35 \%$ & 3 \\
\hline 29 & $5 \%$ & $50 \%$ & $45 \%$ & 2 \\
\hline 30 & $40 \%$ & $30 \%$ & $30 \%$ & 3 \\
\hline
\end{tabular}

Se añade al final de este artículo, como anexo I, el texto con el que los alumnos realizaron la prueba. 
Aunque el grueso de la prueba está realizado en alumnos de nivel medio, 90, y sólo 20 alumnos de nivel avanzado; podemos observar desde un análisis comparativo que ambos grupos comenten los mimos errores o presentan las mismas dudas en el mismo item.

Es significativa la confusión que se produce en el uso que hace el alumno del aspecto flexivo. Hay casos en que se produce una frecuencia casi equivalente de las tres opciones, esto desde nuestro punto de vista es debido a que el enunciado carece de un marcador temporal y que el alumno, por tanto, no tiene clara la teoría ni el uso del aspecto verbal, unido esto a un bajo dominio pragmático de la lengua. Se puede entender la confusión entre perfecto simple y compuesto, hay regiones de ámbito hispánico donde predomina una sobre la otra o se excluye una a otra, pero resulta difícil la alternancia de las tres forma en un mismo item: ejemplo 5 del texto presentado: [5]He pensado mucho en lo que te [6]dije. En este caso dependería de que el emisor del enunciado lo viera como la narración de un hecho o que lo presentara como algo que ocurre en el pasado pero que tiene relación con el presente (pret. perfecto) que es la opción inicial del texto propuesto. Sin embargo, en ningún caso es la descripción de algo habitual ni una descripción del ambiente en que suceden los hechos.
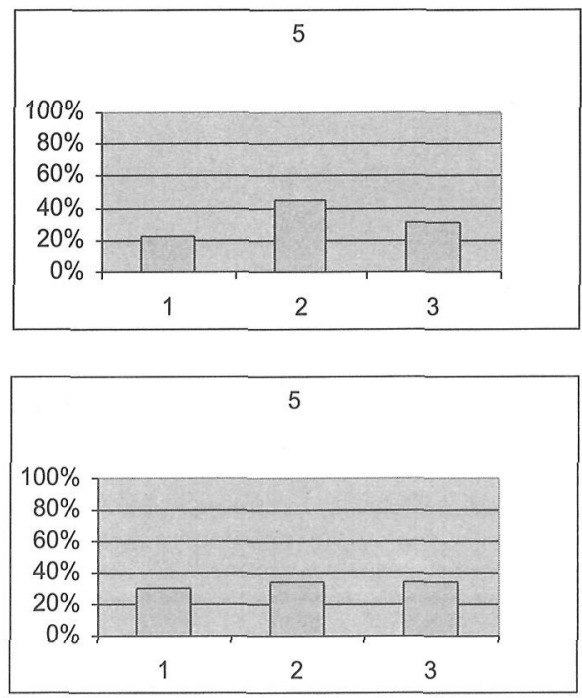

El alumno puede confundir la descripción del ambiente, aunque sea psicológico, con la relación que se establece de lo narrado con el presente, por ejemplo en el caso [22]: no he querido dejar de pensar en ella desde el sábado por la noche. Para el emisor del enunciado, lo importante aquí no es que todos los días haya pensado en ella, sino que es un hecho presente en la enunciación. En este caso, un alto porcentaje de los encuestados optó por la primera opción, el imperfecto. 
Nivel medio

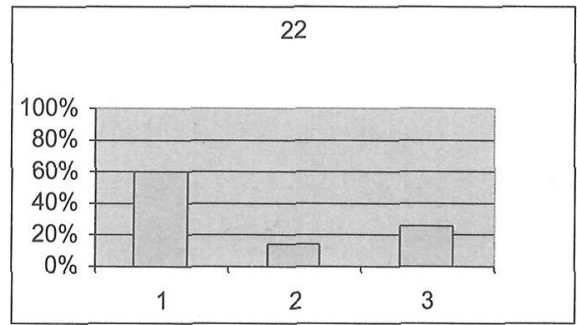

Nivel avanzado

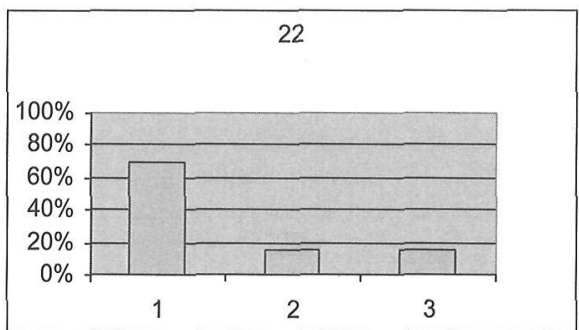

En el caso [27] ocurre lo mismo: Lo sabía de sobra, pero [27]no he podido quedarme de brazos cruzados esta semana.

Nivel medio

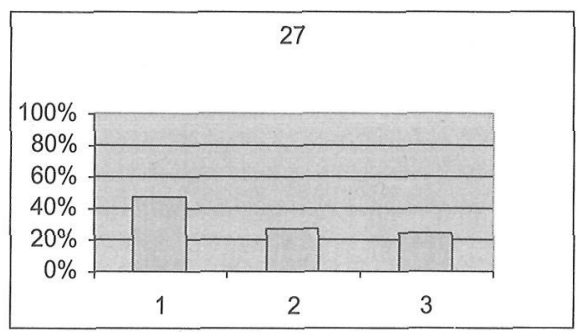

Nivel avanzado

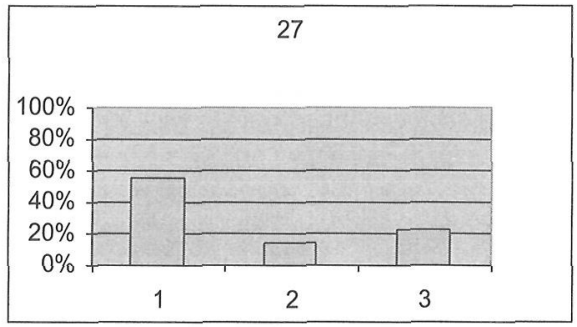


Desde el punto de vista de máxima confusión, la última opción es la más igualada: No sé que resultará de esto pero lo intenté y he tenido la corazonada de que va a ir por buen camino. Desde el punto de vista del emisor se presenta como un hecho que afecta al presente de la enunciación, pero en ambas encuestas el porcentaje está muy igualado en las tres opciones.

Nivel medio

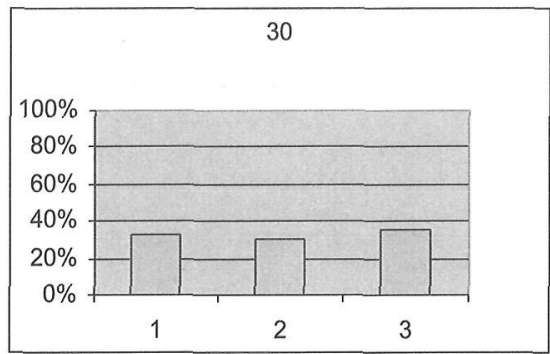

Nivel avanzado

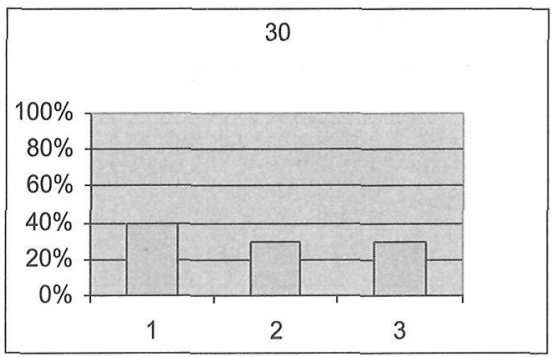

\section{CONCLUSIÓN}

En conclusión, resulta difícil explicar el aspecto sólo en relación con los marcadores temporales. Como consecuencia de ello, el alumno comete menos errores en la medida en que el verbo que hay que sustituir está más próximo a los conectores pero realiza muchos más errores cuando el discurso continua y la proximidad del conector es menor.

Todo lo anterior nos hace pensar que los ejercicios, que plantean los libros de texto en los niveles en que se explica el aspecto, deberían diseñar los supuestos, en los que este se presenta, sin el anclaje de un marcador temporal. De no ser así, la dificultad que supone al alumno extranjero distinguir los matices que presenta el aspecto en español fuera de los ejercicios propiamente realizados ad hoc es mucho mayor, ya que establecen una conexión entre conector y aspecto que no es real. Si bien en un primer momento ayuda a sistematizar el uso del aspecto la utilización de los conectores, creemos que posteriormente lo dificulta.

Esto nos lleva por último a pensar que a la hora de realizar libros de texto de E/LE, habría que plantearse un método gramatical comunicativo en algunos caso, más que el modelo comunicativo simplemente. 


\section{ReFLRLNCLAS BibliogRÁFICAS:}

Baralo, M (1999): La adquisición del español como lengua extranjera. Madrid. Ed. Arco/Libros

De Miguel, F. (1999): «El aspecto léxico» en Gramática descriptiva de la lengua españala. Madrid. Ed. Espasa

García Santa-Cecilia, A. (1995): El curriculo de español como lengua extranjera. Madrid, Ed. Edelsa.

Gustos Gisbert, J.M. (1995): «La temporalidad en español: análisis intencional», LEA, XVII, 2, págs. 14-166

Martínez Bazán, A. (1994): «Análisis transversal del uso de los tiempos indefinido/imperfecto por estudiantcs holandeses de español L2) en Foro Hispánico 6, Revista Hispánica de los Países Bajos, Ámsterdam, Ediciones Rodopi.

Matte Bon, F. (1995): Gramática comunicativa del español. Madrid. Ed. Edelsa

Muñoz, Carmen (Editora) (2000): Segundas lenguas. Adquisición en el aula. Barcelona, Ariel Lingüística.

Reyes, G. (1990):»Tiempo, modo, aspecto e intertextualidad», REL, 20, 1, págs. 17-53.

\section{MANUALES:}

AAVV (1999): Español lengua extranjera, Planet@ ELE, Libro del Alumno y Libro de Referencia Gramatical: Fichas y Ejercicios, Madrid, Edt. Edelsa.

AAVV (2003): Aula, curso de español 2. Barcelona. Difusión.

AAVV (2003): Prisma A2. Madric. Edinumen

Álvarez Martínez, $\mathrm{M}^{\mathrm{a}} \mathrm{A}$ (cood.) (2000): Sueña ELE, Madrid, Anaya ELE.

\section{ANEXO I}

El fin de semana pasado [l]IR (YO). de cena con unos amigos aunque [2]PE NSAR.(YO).. ir a la playa, al final [3]DECIDIR(YO). ir con ellos y después [4]SALIR (NOSOTROS). a tomar una copa. [5]PENSAR (YO).

.... mucho en to que te [6]DECIR (YO)............... no [7]BEBER (YO).

de la cucnta. No te preocupes, te lo [8]PROMETER (YO). . más (YO) a hacerlo. [10]SABER (YO). (ÉL)...... mal.

Cuando [12]SALIR (NOSOTROS) .. que el alcohol me [11]SENTAR TRAR (NOSOTROS). del restaurante nos [13]ENCON...... con una amiga, [15]DECIDIR (NOSOTROS). DAR (ELLA). nos [16]QUEDAR (NOSOTROS). no [18]TENER (YO). con ellas. [17]SABER (YO). final mismo, me [20]GUSTAR (YO). ninguna opción pero [19]VOLVER $(\mathrm{YO})$ a caer en lo [22]QUERER (YO) hace falta, me lo [23]ADVERTIR (TÚ)................, que no [24]TENER (YO)..... y no [21]PODER (YO). .. remediarlo; no ninguna opción y que me [25]EMPEÑARSE (YO)... en algo que es imposible Lo [26]SABER (YO). de sobra, pero no [27]PODER (YO). quedarme de brazos cruzados esta semana y la [28]LLAMAR (YO)........................ para quedar a tomar un cafć. No sé que resultará de esto pero lo [29]INTENTAR (YO).. [30]TENER (YO). la corazonada de que va a ir por buen camino. 


\begin{abstract}
ANEXO II
El fin de semana pasado [1] fui de cena con unos amigos aunque [2]pensaba ir a la playa, al final [3]decidí ir con ellos y después [4]salimos a tomar una copa. [5]He pensado mucho cn lo que te [6]dije: no [7] he bebido más de la cuenta. No te preocupes, te lo [8]he prometido y no [9] he vuelto a hacerlo. [10]Sabía que el alcohol me [11]sentaba mal.

Cuando [12] salimos del restaurante nos [13] encontramos con Mónica que [14]había quedado con una amiga, [15]decidimos acompantarla y al final nos [16]cuedamos con ellas. [17]Sabía que no [18]tenía ninguna opción pero [19] he vuelto a caer en lo mismo, me [20]gustaba y no [21] he podido remediarlo; no [22] he querido dejar de pensar en ella desde el sábado por la noche. No hace falta, me lo [23]has advertido, que no [24]tenía ninguna opción y que me [25] he empañado en algo que es imposible. Lo [26] sabía de sobra, pero no [27] he podido qucdarme de brazos cruzados esta semana y la [28] he llamado para quedar a tomar un café. No sé que resultará de esto pero lo [29]intenté y [30]he tenido la corazonada de que va a ir por buen camino.
\end{abstract}

